\title{
Study of Autoregressive (AR) Spectrum Estimation Algorithm for Vibration Signals of Industrial Steam Turbines
}

\author{
Junyou Huang \\ Sichuan Information Technology College \\ scgyhjy@gmail.com
}

\begin{abstract}
Spectral analysis of the vibration signals of industrial steam turbines provides efficient reference for the characterization and discrimination of turbine faults. Conventional power spectrum estimation methods often exhibit contradiction between variance performance and resolution, leading to poor estimation results. In this study, we investigated Levision-Durbin recursive algorithm, Burg algorithm and periodogram power spectrum estimation algorithm, and also chose Akalke Information Criterion (AIC) to identify the optimal order p. Based on $M A T L A B$, we wrote a simulation program for Autoregressive (AR) spectrum estimation algorithm and designed a graphic user interface, formulating the AR spectrum estimation algorithm program for vibration signals of industrial steam turbines. After field measurement of a steam turbine with sampling number of 400 and frequency of $256 \mathrm{~Hz}$, as well as order of 10 and 80, simulation was performed. It was demonstrated that AIC provides efficient reference for the identification of proper order. With the optimal order, AR spectrum estimation algorithm produces good variance performance and resolution, providing reference for the spectral analysis of vibration signals of industrial steam turbines.
\end{abstract}

Keywords: Steam turbine, Vibration signal, Autoregressive (AR) model, Power spectrum estimation, MATLAB

\section{Introduction}

The power spectral density (PSD) of signals is important for the signal analysis and identification. PSD-related power spectrum estimation technologies are widely employed in areas like radar, sonar, audio and fault diagnosis [1]. Conventional power spectrum estimation is often constrained by the Discrete Fourier Transform (DFT), leading to defects that prohibit high resolution and accuracy, which are required in some cases [2]. These defects include leakage error, aliasing error, low resolution, and unsuitability for short data processing, rough spectral lines, violent fluctuation, and difficulty in fitting smooth curves [3]. In modern spectrum estimation, parameter model is first estimated by observing data, and then the power spectrum of signal is estimated by calculating the output power of the parameter model [4]. Autoregressive (AR) model, an all-pole model that can effectively describe the peaks of narrow-band power spectra, is a typical model in modern spectrum estimation [5]. With an order great enough, AR model can describe a stationary random sequence in a relatively precise fashion [6].

Steam turbine is a type of rotating machinery widely used in industrial field. The conditions of turbines influence both their normal operations and the production safety and economic benefits. When faults occur to a turbine, the spectral energy distribution of its vibration signal often changes. Therefore, spectral analysis of the vibration signal can provide efficient reference for the fault diagnosis [7]. In this study, we investigated AR power 
spectrum estimation algorithm, performed comparative analysis using MATLAB simulation and designed a graphical user interface (GUI). As a result, a set of simulation programs, which offers flexible signal generation, sampling frequency selection and parameter setup and provides estimation result comparison and analysis between different parameter setups, were designed. On the basis of these programs, we analyzed the performances of different algorithms using actual vibration signal of an industrial steam turbine, hoping to provide reference for the spectral analysis of vibration signals of steam turbines.

\section{Algorithms}

\subsection{Periodogram Power Spectrum Estimation Algorithm}

Periodogram method is to perform Fast Fourier Transform (FFT) to the definite number of samples $x(n)=\{x(0), x(1), \cdots, x(N-1)\}$ of observed signal directly for power spectrum estimation[8]. The N-periodogram of $x(n)$ can be calculated by Formula (1).

$$
I_{N}(k)=\left.I_{N}(\omega)\right|_{\omega=\frac{2 \pi}{N} k}=\frac{1}{N}|X(k)|^{2}
$$

Where, $\mathrm{N}$ is the length of data and $\mathrm{X}(\mathrm{k})$ is the discrete Fourier transform of signal sequence $\mathrm{x}(\mathrm{n}) . \mathrm{X}(\mathrm{k})$ is calculated through FFT and it should be called in the format of $\mathrm{Xk}=\mathrm{fft}(\mathrm{xn}$, NFFT). The algorithm can be described by the flowchart in Figure 1.



Figure 1. Periodogram Spectrum Estimation Algorithm for Steam Turbine

The code for this process is as below.

$\operatorname{mid}=\operatorname{sum}(x n) /$ length $(x n) ; x n=x n-m i d ; \%$ Remove DC component

$\mathrm{N}=$ length (xn);

fs $=256 ;$ NFFT $=1024 ; \%$ Configure sampling frequency and FFT number

$\mathrm{Xk}=\mathrm{fft}(\mathrm{xn}, \mathrm{NFFT}) ; \%$ Perform FFT with number of NFFT for Xn 
xpsd $=X k . * X k / N ; \%$ Calculate power spectrum

$\operatorname{pmax}=\max (\mathrm{xpsd})$;

$\mathrm{xpsd}=\mathrm{xpsd} / \mathrm{pmax}$;

xpsd $=10 * \log 10(x p s d+0.000001) ; \%$ Normalize the power spectrum and convert it into logarithmic spectrum

$\mathrm{k}=0: 1:(\mathrm{NFFT} / 2-1)$;

$\mathrm{f}=\mathrm{fs} * \mathrm{k} / \mathrm{NFFT} ; \%$ Convert the discrete frequency to actual frequency

plot(f,xpsd(1: NFFT/2)); grid on;xlabel('f/Hz'); ylabel('PSD/dB'); \%Draw the power spectrum

\subsection{AR Power Spectrum Estimation Algorithm}

2.2.1. Algorithm Flow: AR power spectrum estimation algorithm is established on the basis of performing linear prediction modeling to the signal sequence $\mathrm{x}(\mathrm{n})$ to be estimated. The sequence is treated as a white noise sequence with mean of 0 and variance of $\sigma_{w}^{2}$, and it is generated by a Linear Time Invariant (LTI) system with system function of H(z). It can be expressed by the difference equation below.

$$
x(n)=-\sum_{k=1}^{p} a_{k} x(n-k)+w(n)
$$

This model is denoted by $\mathrm{AR}(\mathrm{p})$, and the system transition function $\mathrm{H}(\mathrm{z})$, as expressed below, is a p-order AR model.

In power spectrum estimation, if the observed data $\mathrm{x}(\mathrm{n})$ is a stationary random process, the input $\mathrm{w}(\mathrm{n})$ of the system can be considered stationary[9]. According to the response theory of linear systems to stationary random signals, the power spectrum of the observed data can be expressed by Equation (4).

$$
P_{x}(\omega)=\sigma_{w}^{2}\left|H\left(e^{j \omega}\right)\right|^{2}=\frac{\sigma_{w}^{2}}{\left|1+\sum_{k=1}^{p} a_{k} e^{-j \omega k}\right|^{2}}
$$

The denominator of Equation (4) can be calculated through FFT. In this algorithm, a signal prediction model is established to signal sequence $\mathrm{x}(\mathrm{n})$ through linear prediction to predict the data outside the sampling intervals, so that spectral leakage in conventional spectrum estimation algorithms, caused by the window truncating of data, is prevented. This way, the estimation result is improved comparing with conventional algorithms. Figure 2 presents the flow of this algorithm. 


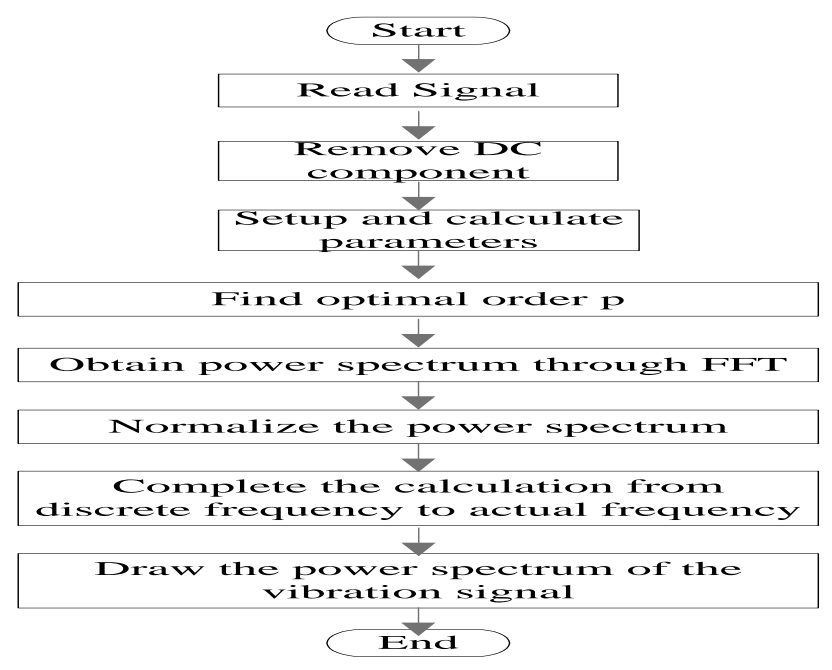

Figure 2. AR Power Spectrum Estimation Algorithms

2.2.2. Identification of Optimal Order through AIC: To use the AR power spectrum estimation algorithm for vibration signals of industrial turbines, the identification of the model's order $\mathrm{p}$ is crucial. In practical application, there exist optimal orders, which can be identified according to the Akalke Information Criterion (AIC).

$$
A I C(p)=\ln \left[\sigma_{p}^{2}\right]+\frac{2(p+1)}{N}
$$

Where, $\mathrm{N}$ is the length of data $\mathrm{x}(\mathrm{n})$, and $\sigma_{p}^{2}$ is the prediction error rate of AR model with order $\mathrm{p}$ [10]. When a model's AIC function has the minimal value, it is the suitable model. Based on AIC and combining aryule function, the prediction error rate $\sigma_{p}^{2}$ is calculated. The following program is designed to calculate and plot the AIC (p) curve for the convenience of deciding the optimal order $\mathrm{p}$.

2.2.3. L-D recursive Algorithm-based AR Spectrum Estimation: Assume that the observed data $\mathrm{x}(\mathrm{n})$ is obtained by stimulating an all-pole linear time invariant system $\mathrm{H}(\mathrm{z})$ using a zero-mean white noise sequence $\mathrm{W}(\mathrm{n})$ with mean square error of $\sigma_{w}^{2}$ [11-12]. On the premise of keeping the mean square error of forward prediction minimal, Levision-Durbin (LD) recursive algorithm acquires the auto-correlation function of the observed data, obtains the model's parameters through the recursive property of Yule-Walker (Y-W) function, and then calculates the estimated power spectrum using Equation(4) [13]. First, the predicting coefficients $\left\{a_{m}(k)\right\}=a_{1}(1)$ and $\sigma_{w 1}^{2}$ when the order $\mathrm{m}=1$ are calculated; then the coefficients $a_{2}(1), a_{2}(2)$ and $\sigma_{w 2}^{2}$ when the order $\mathrm{m}=2$ are calculated; in the same manner coefficients are calculated until $a_{p}(1), a_{p}(2), \ldots a_{p}(p)$ and $\sigma_{w p}^{2}$ for $\mathrm{m}=\mathrm{p}$, when $\sigma_{p}^{2}$ satisfies the requirements for precision. The recursive formula is as below.

$$
\begin{aligned}
& a_{m}(m)=-\frac{R(m)+\sum_{k=1}^{m-1} a_{m-1}(k) R(m-k)}{E_{m-1}} \\
& a_{m}(k)=a_{m-1}(k)+a_{m}(m) a_{m-1}(m-k), k=1,2, \ldots m-1 \text { (7) }
\end{aligned}
$$




$$
E_{m}=\sigma_{w m}^{2}=\left[1-\left|a_{m}(m)\right|^{2}\right] E_{m-1}=R(0) \prod_{k=1}^{m}\left[1-\left|a_{k}(k)\right|^{2}\right]
$$

The computational labor of L-D algorithm is in the magnitude of $p^{2}$. With parameters $\mathrm{AR}(0)$ and $\mathrm{AR}(1)$ of the model as initial conditions, $\mathrm{AR}(2)$ is calculated, and then $\mathrm{AR}(3)$ to $\mathrm{AR}(\mathrm{P})$ in likewise manner. After the iterative computation, the parameters for the low-order models are also obtained [14]. In MATLAB, this algorithm can be realized with pyulear function as the core. The pyulear function should be called in the format of $x p s d=p y u l e a r(x n$, $\mathrm{p}$,NFFT), where xpsd indicates the calculated power spectrum of the signal, $\mathrm{xn}$ is the signal sequence to be estimated, $\mathrm{p}$ is the order of AR model, and NFFT is the number of Fast Fourier Transform (FFT), which must be greater than or equal to the sampling number of the signal. The following program details the L-D recursive algorithm.



2.2.4. Burg Algorithm-based AR Spectrum Estimation: The basic idea of Burg algorithm is to use linear predictor to directly calculate the total mean square error of the forward and backward predictions of the observed data, keep the total mean square error minimal in order to estimate the reflection coefficient, and then find the optimizing parameters for AR model through the recursive formula of L-D algorithm [15]. For $\mathrm{N}$ observed data $\mathrm{x}(0), \mathrm{x}(1), \ldots, \mathrm{x}(\mathrm{N}-$ 1), the algorithm executes in the flow presented by Figure 3.

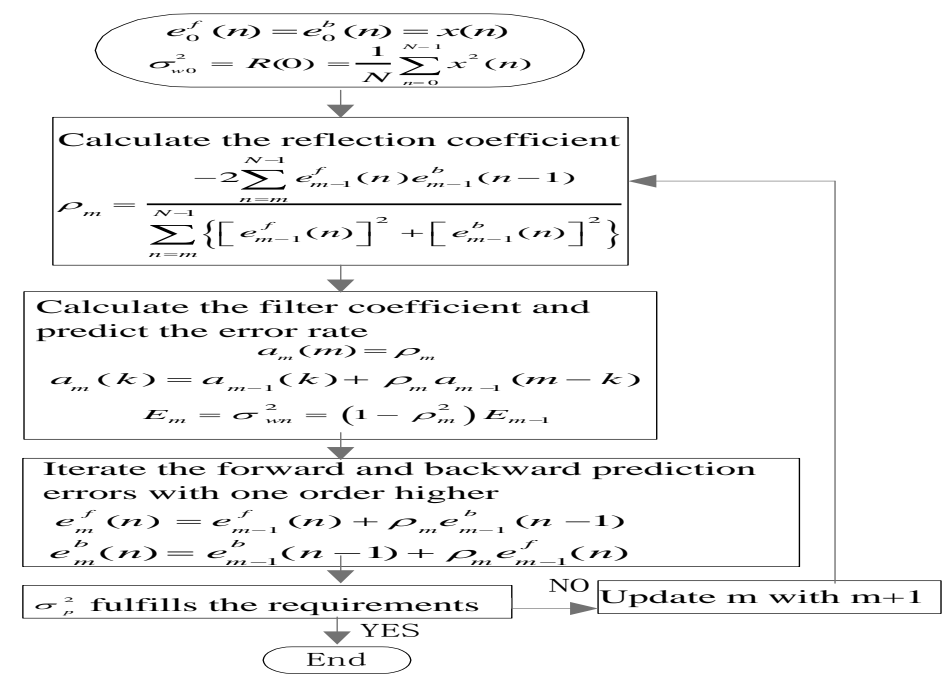

Figure 3. Flowchart for Burg Algorithm-Based AR Spectrum Estimation 
In MATLAB, function pburg should be called for this process. The calling of pburg function should be in the format of xpsd=burg (xn, p,NFFT), similar to the calling of L-D recursive algorithm, and the program code is as below.

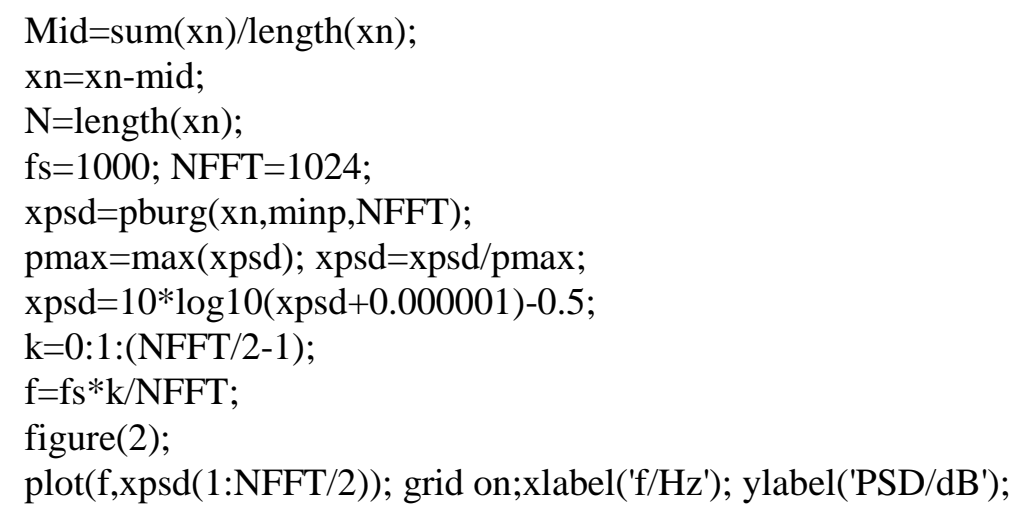

\section{Design of GUI and Program}

The design of the graphic user interface (GUI) includes the arrangement of components, the compiling of properties and the compiling of the callback function. In MATLAB, typing command 'guide' can create an initial GUI page. With the appearance editing function of GUIDE, necessary controls can be drew to the interface with properties configured. A single click of the 'Run' button in the toolbar of GUIDE will activate the graphic user interface.

\subsection{Signal Selection Function}

The realization of signal selection function is described with signal source $4-256 \mathrm{~Hz}$ (as presented in Figure 4) as an example.



Figure 4. Signal Source of $256 \mathrm{~Hz}$

In MATLAB, type 'guide main.fig' in Command Window and double click the 'vibration signal sample selection' module, the property compiler will appear. Set the value of 'string' as 'vibration signal sample selection' and 'tag' as 'signalselect', single click the 'vibration signal sample selection' module and choose the 'callback' button in 'View callbacks' of the pull down list, and type the following code:

[FileName,PathName] = uigetfile('*.txt'); datafile $=$ fullfile $($ PathName,FileName); 


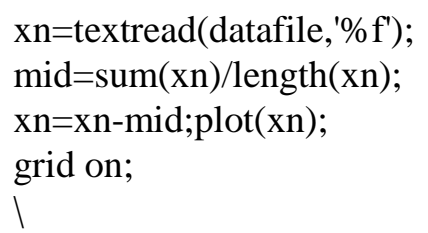

Then, save the code and execute, signal source $4-256 \mathrm{~Hz}$ is selected.

\subsection{Parameter Display Function}

The function of parameter display should be configured in the "vibration signal sample selection' module. The main program for this function is as below.

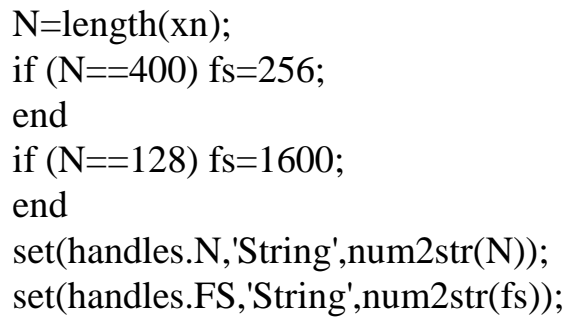

\subsection{Single Selection of Mutual Exclusion}

To detail the realization of this function, assume that during the algorithm selection of AR spectrum estimation, L-D recursive algorithm was chosen. Set the value of 'string' of periodogram method as 'perodogram method' and 'Tag' as 'PER', and set the values of 'Tag' of 'AR model (L-D)' and 'AR model (BURG) as 'ARLD' and 'ARBURG', respectively. Choose the module corresponding to 'AR model (L-D)' and click the 'callback' button in 'View callbacks' of the pull down list, and input the following code:

set(findobj('Tag','PER'),'value',0);

set(findobj('Tag','ARBURG'),'value',0);

Set the value of Object with 'Tag' of 'PER' as 0 , and the value of Object with 'Tag' of 'ARBURG' as 0, then when the 'AR model (L-D)' control is selected for spectrum estimation, periodogram method and AR model (BURG) are chosen for exclusion.

\subsection{Parameter Setup Function}

The realization of parameter setup function is detailed here with the specification of order as an example. In the parameter setup of controls, single click on the SN (specified order) and the AICN (optimal order) modules, and click the 'callback' buttons in 'View callbacks' of the pull down lists, input the following two lines of code respectively.

set(findobj('Tag','AICN'),'value',0);

set(findobj('Tag','SN'),'value',0);

Then, save and execute, the parameter setup function is realized.

\subsection{Power Spectrum Estimation Function}

For the calculation of optimal order for signal source of $4-256 \mathrm{~Hz}$ and L-D recursive algorithm as an example, execute the L-D recursive algorithm-based AR spectrum estimation, and the following program identifies the optimal order. 


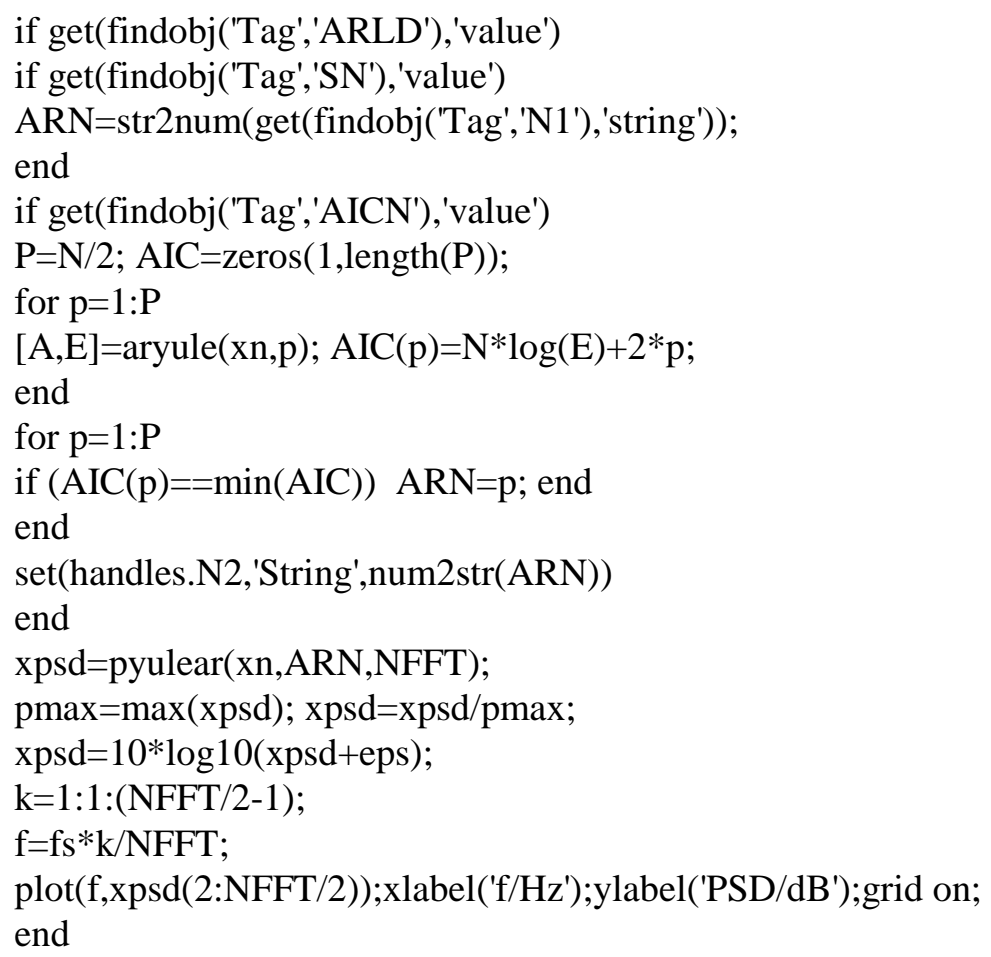

After executing this program, the result as shown in Figure 5 is obtained.

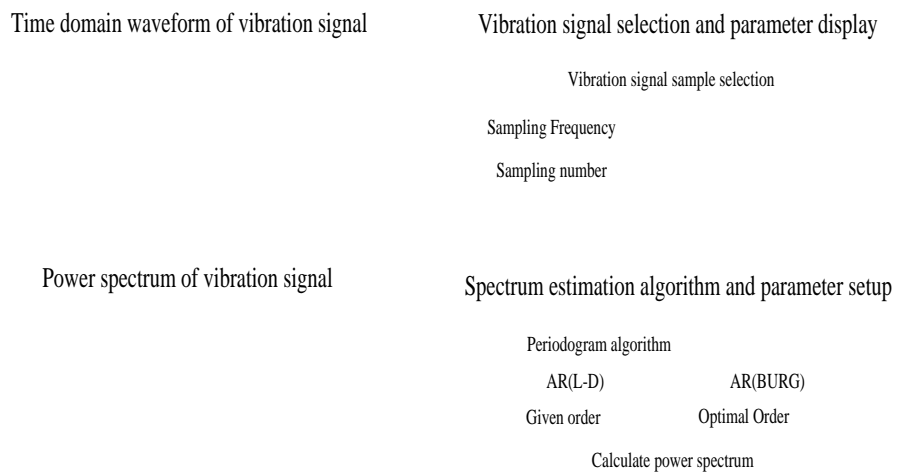

Figure 5. Power Spectrum Estimation Function

\section{Performance Analysis of the Algorithm}

\subsection{Signal Collection}

Through field measurement, vibration signals of an industrial turbine were collected. Electronic vortex sensor with ideal shaft rotating frequency of $50 \mathrm{~Hz}$ was used. During the signal collection, for fixed-frequency sampling, the highest frequency was $256 \mathrm{~Hz}$, while for frequency multiplication sampling the highest sampling frequency was the 128 times of the rotating rate. 
In this study, we investigated the variance performance and resolution of periodogram method and AR spectrum estimation algorithm, compared their performances with different parameter setup, chose the optimal algorithm and related parameters and applied them to the analysis of the measured signals. The signals were obtained through fixed-frequency sampling with frequency of $256 \mathrm{~Hz}$ and 32 times-frequency multiplication sampling. The time domain waveform of the vibration signal collected with sampling frequency of $256 \mathrm{~Hz}$ and sampling number of 400 is presented in Figure 6.

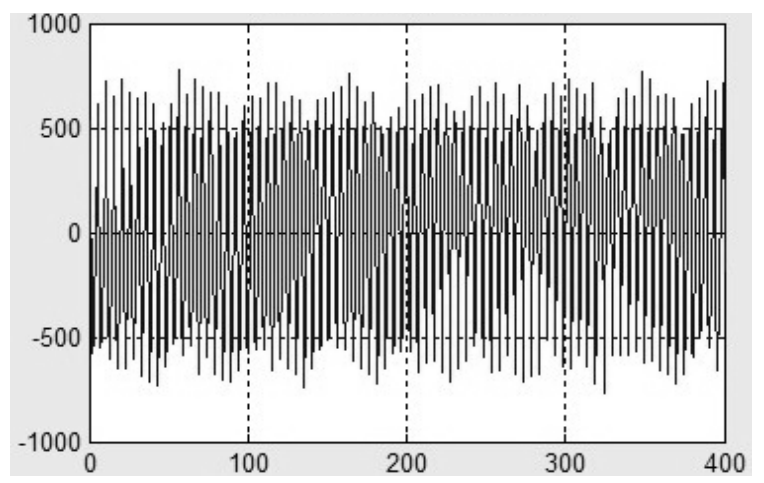

Figure 6. Time Domain Waveform of Measured Vibration Signal

The time domain waveform of the vibration signal collected by 32 times-frequency multiplication sampling with frequency of $f_{s}=50 \times 32=1600 \mathrm{~Hz}$ and sampling number of 128 is presented in Figure 7.

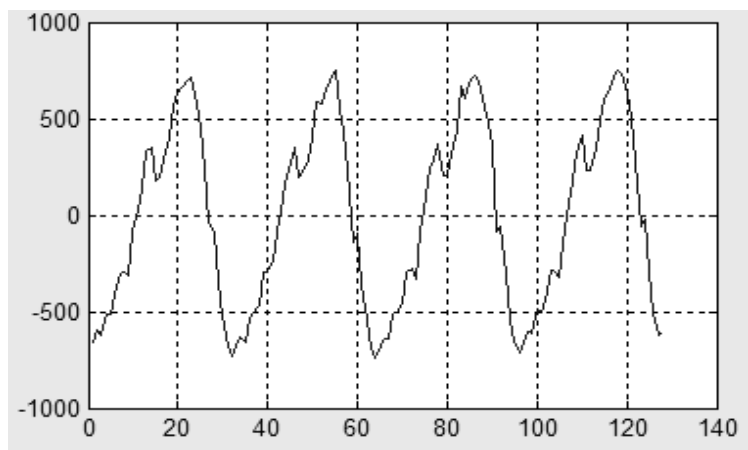

Figure 7. Time Domain Waveform of Measured Vibration Signal with Sampling Number of 128

With the waveforms in Figure 6 and 7, it is difficult to analyze the characteristics of the vibration signals accurately from the perspective of time domain and to discover and locate faults. Therefore, the aid from power spectrum analysis tools is needed.

\subsection{Result and Analysis of Periodogram Estimation}

With sampling number of 400 and sampling frequency of $256 \mathrm{~Hz}$, the simulation result of periodogram method is as shown in Figure 8. 


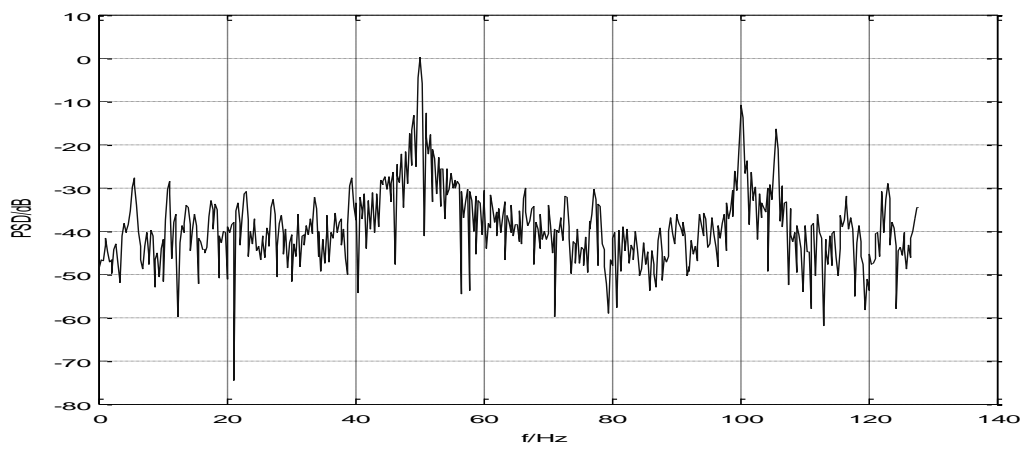

Figure 8. Estimation Result of Periodogram Method

It can be seen from the result that the spectrum obtained by periodogram method has good resolution and sharp peaks. However, the variance performance is poor, and the spectrum shows violent fluctuations, making it hard to ensure the accuracy of spectral analysis. In addition, the spectrum has poor tolerance to noises. Such contradiction between the variance and resolution makes the application of periodogram method in turbine vibration signal analysis limited. When it is impossible to collect and process longer vibration signal data because of the constraint of real-time performance and processing speed, such limitation is particularly obvious. In such case, the random sequence $x(n)$ is treated as periodic extension of a section of finite sequence, thus causing spectral leakage and affecting the spectrum estimation performance. It seems that the smaller the sampling number, i.e. the shorter the windowing length, the more obvious the spectral leakage.

\subsection{Results of L-D recursive algorithm- and Burg algorithm-based AR spectrum estimations}

With sampling number of 400 , sampling frequency of $256 \mathrm{~Hz}$, and optimal order of 52 , the simulation result of L-D recursive algorithm-based AR spectrum estimation is as shown in Figure 9.

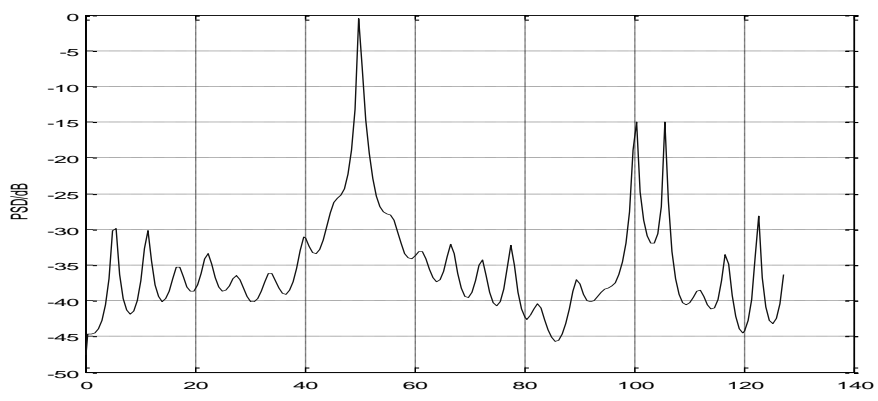

Figure 9. L-D Recursive Algorithm-Based AR Spectrum Estimation Result

It can be seen that the spectrum has good resolution, sharp peaks and accurate harmonic location. The fluctuation is not violent, indicating good variance performance, and the tolerance to noises is improved. The simulation shows that the L-D recursive algorithm-based AR spectrum estimation is suitable for vibration signals of steam turbines. The simulation result of Burg algorithm-based AR spectrum estimation is as shown in Figure 10. 




Figure 10. Burg Algorithm-Based AR Spectrum Estimation Result

It can be seen that the spectrum has good resolution and sharp peaks. The fluctuation is relatively violent, which means the variance performance is less satisfying, yet the harmonic components can be located from the noises. This simulation result demonstrates that Burg algorithm-based AR spectrum estimation is also suitable for vibration signals of steam turbines.

\subsection{Comparison between Periodogram Method and AR Spectrum Estimation}

Comparing Figure 8, 9 and 10, it can be found that AR model has comparable frequency resolution with periodogram method but much better variance performance. Through comparative analysis, we found that with proper selection of order, AR spectrum estimation algorithm can ensure both good resolution and variance performance, with much better estimation result. In the analysis of vibration signals of steam turbines, AR model can perform relatively accurate harmonic analysis even with short sampling data, overcoming the limitation of conventional algorithms.

\subsection{Comparison between L-D recursive Algorithm and Burg Algorithm}

It can be seen from Figure 9 and 10 that comparing with L-D recursive algorithm, Burg algorithm has better resolution, slightly displaced frequency and less satisfying variance performance [16]. Since when performing auto-correlation sequence estimation, L-D recursive algorithm assumes the data outside the range of $0 \sim \mathrm{N}-1$ as 0 , the estimated power spectrum are more smooth, with peaks less sharp than those estimated by Burg algorithm, and the displacement is greater. In practical application, L-D recursive algorithm-based AR spectrum estimation is more suitable for the estimations with higher requirement of variance performance, whereas Burg algorithm-based AR spectrum estimation is more suitable for estimations with higher requirement of resolution.

\subsection{Influences of Order on AR Spectrum Estimation Result}

With L-D recursive algorithm-based AR spectrum estimation, simulation analyses are performed to the signal in Figure 11, with given order of 10 and 80 and identified optimal order of 52. The estimated power spectra are presented in Figure 11, 12 and 13, respectively. 


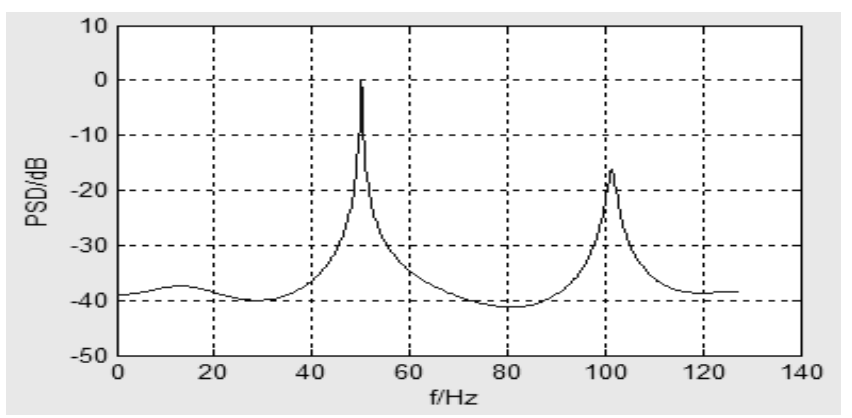

Figure 11. Power Spectrum Estimated with Order of 10

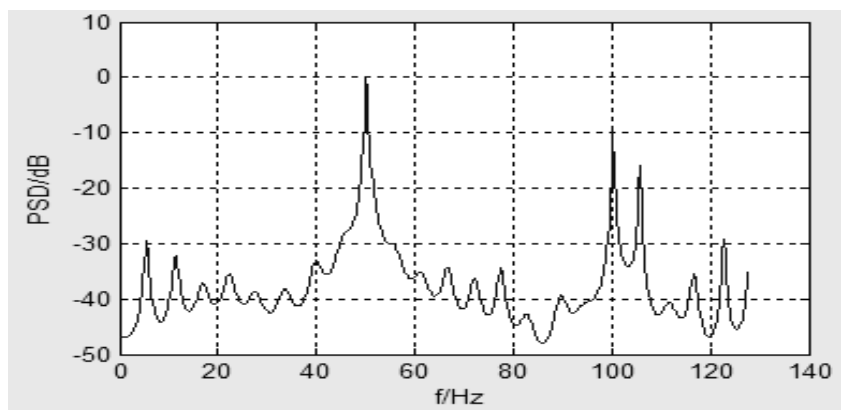

Figure 12. Power Spectrum Estimated with Optimal Order of 52

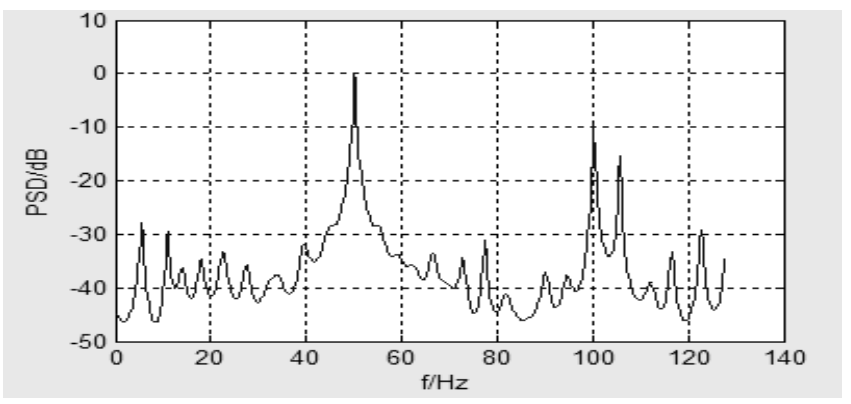

Figure 13. Power Spectrum Estimated with Order of 80

Comparing Figure 11, 12 and 13, the following findings were made. With overly low order $\mathrm{p}$, the estimated spectrum is too smooth, which reduces the resolution and causes some harmonic components to be unidentifiable. With overly high order $\mathrm{p}$, noise and other interference signals would be prominent in the spectrum as useful signals, creating false peaks and increasing the computational labor. With optimal order, the waveform is smoother, peaks sharper, and resolution and variance performance more balanced. For the processing and analysis of vibration signals, AR spectrum estimation algorithm shows good balance between resolution and variance performance.

\section{Conclusions}

Based on field measurements of vibration signals of an industrial steam turbine, comparative analysis was performed between AR spectrum estimation and conventional spectrum estimation algorithms. Through theoretical study, algorithm analysis and actual simulation, we came to the following conclusions. Conventional spectral estimation algorithm 
exhibits contradiction between resolution and variance performance. With smaller sampling number, the algorithm shows poor performance for vibration signals of turbines, thus it has some limitation in application. For the spectrum estimation of turbine vibration signals, conventional algorithms are unable to perform short data spectral analysis in practical application, whereas this limitation is o overcome by AR spectrum estimation algorithm. Satisfying harmonic analysis result can be obtained by AR model. In AR spectrum estimation, the order of the model greatly influences the estimation result for turbine vibration signals, thus it is an important parameter of the model. In the spectrum estimation of turbine vibration signals, AIC provides good theoretical basis for the identification of optimal order.

\section{References}

[1] I. Sadinezhad and V. G. Agelidis, "Slow sampling on-line harmonics/interharmonics estimation technique for smart meters", Electric Power Systems Research, vol. 81, no. 8, (2011), pp. 1643-1653.

[2] M. D. Ortigueira and J. M. Tribolet, "Global versus local minimization in least-squares AR spectral estimation”, Signal Processing, vol. 7, no. 3, (1984), pp. 267-281.

[3] T. Al-ani, F. Cazettes, S. Palfi and J.-P. Lefaucheur, "Automatic removal of high-amplitude stimulus artefact from neuronal signal recorded in the subthalamic nucleus”, Journal of Neuroscience Methods, vol. 198, no. 1, (2011), pp. 135-146.

[4] M. Rezazadeh Mehrjou, N. Mariun, M. Hamiruce Marhaban and N. Misron, "Rotor fault condition monitoring techniques for squirrel-cage induction machine-A review", Mechanical Systems and Signal Processing, vol. 25, no. 8, (2011), pp. 2827-2848.

[5] R. Chen, X. Zhao, Z. Wang, H. Jiang and X. Bian, "Experimental study on dynamic load magnification factor for ballastless track-subgrade of high-speed railway", Journal of Rock Mechanics and Geotechnical Engineering, vol. 5, no. 4, (2013), pp. 306-311.

[6] E. Derya Übeyli and İ. Güler, "Selection of optimal AR spectral estimation method for internal carotid arterial Doppler signals using Cramer-Rao bound", Computers \& Electrical Engineering, vol. 30, no. 7, (2004), pp. 491-508.

[7] A. Abouhnik and A. Albarbar, "Wind turbine blades condition assessment based on vibration measurements and the level of an empirically decomposed feature", Energy Conversion and Management, vol. 64, (2012), pp. 606-613.

[8] R. Zolfaghari, Y. Shrivastava and V. G. Agelidis, "Evaluation of windowed ESPRIT virtual instrument for estimating Power Quality Indices”, Electric Power Systems Research, vol. 83, no. 1, (2012), pp. 58-65.

[9] B. Muruganatham, M. A. Sanjith, B. Krishnakumar and S. A. V. Satya Murty, "Roller element bearing fault diagnosis using singular spectrum analysis", Mechanical Systems and Signal Processing, vol. 35, no. 1-2, (2013), pp. 150-166.

[10] K. Vedavathi, K. Srinivasa Rao and K. Nirupama Devi, "Unsupervised learning algorithm for time series using bivariate AR(1) model”, Expert Systems with Applications, vol. 41, no. 7, (2014), pp. 3402-3408.

[11] R. Giacometti, M. Bertocchi, S. T. Rachev and F. J. Fabozzi, "A comparison of the Lee-Carter model and AR-ARCH model for forecasting mortality rates", Insurance: Mathematics and Economics, vol. 50, no. 1, (2012), pp. 85-93.

[12] E. G. Gamaly, "The physics of ultra-short laser interaction with solids at non-relativistic intensities", Physics Reports, vol. 508, no. 4-5, (2011), pp. 91-243.

[13] T. Rosenkranz and H. Puder, "Integrating recursive minimum tracking and codebook-based noise estimation for improved reduction of non-stationary noise", Signal Processing, vol. 92, no. 3, (2012), pp. 767-779.

[14] M. A. Altınkaya, H. Deliç, B. Sankur and E. Anarım, "Subspace-based frequency estimation of sinusoidal signals in alpha-stable noise", Signal Processing, vol. 82, no. 12, (2002), pp. 1807-1827.

[15] M. G. Atwia, M. M. Abu-Heleika and M. M. El-Horiny, "Hydrogeochemical and vertical electrical soundings for groundwater investigations, Burg El-Arab area, Northwestern Coast of Egypt", Journal of African Earth Sciences, vol. 80, (2013), pp. 8-20.

[16] D. I. Lekhovytskiy and Y. S. Shifrin, "Statistical analysis of 'superresolving' methods for direction-of-arrival estimation of noise radiation sources under finite size of training sample", Signal Processing, vol. 93, no. 12, (2013), pp. 3382-3399. 


\section{Author}

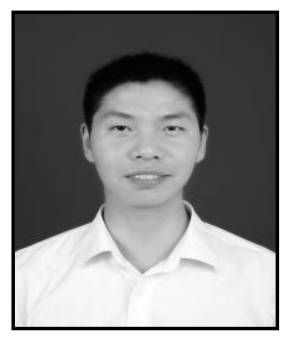

Junyou Huang was born in Sichuan, China. After receiving his bachelor's degree in applied electronic technologies in Chengdu Institute of Information Engineering, he has been a faculty member of Department of Electronic Engineering in Sichuan Information Technology College. $\mathrm{He}$ received his Master's degree in Electronic and Communication Engineering in University of Electronic Science and Technology of China. He is now an associate professor with major research interests in electronic and communication technologies. 